Research Paper

\title{
USP32 promotes tumorigenesis and chemoresistance in gastric carcinoma via upregulation of SMAD2
}

\author{
Ning Dou, Qingqing $\mathrm{Hu}$, Li Li, Qiong Wu, Yandong $\mathrm{Li}^{\bowtie}$, Yong Gao ${ }^{\bowtie}$ \\ Department of Oncology, Shanghai East Hospital, Tongji University School of Medicine, Shanghai 200120, China. \\ $\bowtie$ Corresponding authors: Yandong Li (E-mail: yandongli2017@tongji.edu.cn) \& Yong Gao (E-mail: drgaoyong@tongji.edu.cn).
}

(C) The author(s). This is an open access article distributed under the terms of the Creative Commons Attribution License (https://creativecommons.org/licenses/by/4.0/). See http://ivyspring.com/terms for full terms and conditions.

Received: 2019.12.16; Accepted: 2020.02.29; Published: 2020.03.12

\begin{abstract}
USP32, a member of the ubiquitin-specific proteases family, has been implicated in the development of breast cancer and small lung cancer. However, its biological functions and clinical significance in gastric cancer (GC) remain unclear. In the present study, we reported that knockdown or depletion of USP32 significantly inhibited GC cell proliferation and migration in vitro and in vivo, indicating that USP32 functions as an oncogene in GC. Importantly, results from immunohistochemical staining in a tissue microarray revealed that USP32 was upregulated in GC tissues compared with paracancerous tissues. Further analyses showed that high expression of USP32 was closely related with high T-staging and poor outcomes of GC patients. Mechanistically, USP32 silencing caused a decrease in the expression of SMAD2, which resulted in the inhibitory effects of GC cells on growth, motility, and chemoresistance to cisplatin. Therefore, our findings strongly suggest the involvement of USP32 in GC progression and provide a potential target for future therapy of GC.
\end{abstract}

Key words: USP32; SMAD2; gastric carcinoma; drug resistance

\section{Introduction}

Protein ubiquitylation, one of the posttranslational modifications (PTMs), plays important role in regulating protein degradation, signal transduction, and DNA damage responses [1,2]. Ubiquitylation is a reversible process, and generally ubiquitin was added to substrates by the E1 activating enzymes, E2 conjugating enzymes and E3 ligating enzymes then removed by deubiquitylating enzymes (DUBs) [3]. Deubiquitylating enzymes (DUBs) have been recognized as powerful tools for altering stability, localization, and activity of proteins, which are widely implicated in pathological mechanisms of many human diseases [4-6].

Nearly 100 DUBs are identified in the mammalian genome, and there are several subclasses of DUBs. Structurally, ubiquitin carboxy (C)-terminal hydrolases (UCHs), ubiquitin-specific proteases (USPs), Machado-Joseph disease protein domain proteases (MJDs) and ovarian tumor proteases (OTUs) are papain-like cysteine proteases, while JAB/MPN/Mov34 metalloenzyme (JAMM) domain proteases are zinc-dependent metalloproteases [7]. The USP subfamily, with more than 50 members in humans, has been reported to play pivotal roles in tumors [8-10]. USP28 is identified as a key regulator of cell proliferation in colorectal cancer [11]. USP22 can function as a tumor driver gene via regulating cell cycle [12]. USP46 is selectively recruited by the high-risk HPV and promotes cell proliferation in high-risk HPV caused cervical cancer [13]. On the contrary, USPs can also be recognized as tumor suppressors, such as USP10, USP17 and USP49 have been implicated in several cancer types [14-16].

USP32, a new member of the USP subfamily, is an ancient and highly conserved gene located on 17q23. It is reported to be the origin of Tre2 (USP6) oncogene, and nucleotides 3194-6063 of USP6 mRNA have $97 \%$ sequence identity to USP32 [17]. Some reports have revealed its involvements in cancer. In fact, USP32 exhibits overexpressed in breast cancer and human small cell lung cancer and may serve as an oncogene through promoting cell proliferation and 
tumor metastasis $[18,19]$. However, little is known about the function and expression of USP32 in gastric cancer.

Emerging studies have indicated key roles for ubiquitin modification in transforming growth factor- $\beta$ (TGF- $\beta$ ) signaling [20]. For example, the E3 ligase TRIM33/Ectodermin has been shown to inhibit ubiquitination of SMAD4 [21]. In addition, Smurf2 binds to Smad7 forms a SMAD7-SMURF2 complex which activates and degrades TGF- $\beta$ receptor [22]. In this study, we partially set up a link between USP32 and SMAD2, a key component of TGF $\beta$ signaling pathway, in GC development and progression. We found that USP32 affected the SMAD2 expression partially in an ubiquitin protease dependent manner. Functionally, SMAD2 may mediate the oncogenic effects of USP32 on cell growth, metastasis and drug resistance. Moreover, we also found that USP32 is upregulated in gastric cancer tissues and its expression has strong clinical relevance. Taken together, these results provide a novel insight of USP32/SMAD2 axis in GC progression.

\section{Materials and methods}

\section{Cell culture}

GC cell lines, MGC803 and SGC7901 were purchased from the cell bank of the Chinese Academy of Sciences at Shanghai, China. GC cells were grown in Dulbecco's Modified Eagle's medium (DMEM, Corning, US) containing 10\% fetal bovine serum (FBS) supplemented with penicillin/streptomycin $(100 \mu \mathrm{g} /$ $\mathrm{mL}$ ). The cells were maintained at $37^{\circ} \mathrm{C}$ and $5 \% \mathrm{CO}_{2}$.

\section{Colony formation assay}

GC cells $(2,000$ cells per well) were plated in 6 well plates and incubated for 14-20 days in triplicate. Then colonies were washed with PBS and stained with $0.5 \%$ crystal violet for $15 \mathrm{~min}$. The visible colonies were photographed and counted results were obtained from three independent experiments.

\section{Cell proliferation assay}

For proliferation assay, GC cells $(3,000$ cells per well) were seeded into 96-well culture plates in triplicate and incubated for 5 days at $37^{\circ} \mathrm{C}$ in a humidified incubator with $5 \% \mathrm{CO}_{2}$. Every $24 \mathrm{~h}$ interval, $10 \mu \mathrm{l}$ of Cell Counting kit-8 (CCK-8; Dojindo Laboratories, Japan) was added to each well and incubated at $37^{\circ} \mathrm{C}$ for $1 \mathrm{~h}$. Then the plates were read at 450 nm (SpectraMax M5, Molecular Devices, US). All these experiments were repeated at least three times.

\section{Transwell chamber assay}

For in vitro migration assay, cells (30,000 cells per well) were planted in the upper chambers (Corning,
US) in serum free medium in 24-well plates. The bottom was added with DMEM plus 10\% FBS (fetal bovine serum). After cultured 24-36 h, cells on the undersurface were stained with crystal violet and calculated under microscopy. Each experimental group included three replicates.

\section{Western blotting}

Cells were lysed in RIPA buffer, and protein concentration was measured using a BCA Assay Kit

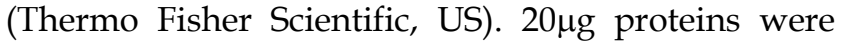
electrophoresed by SDS-polyacrylamide gels and transferred onto a nitrocellulose membrane (Millipore, US). The membrane was then blocked in non-fat milk for $1 \mathrm{~h}$ at room temperature and subsequently incubated with the primary antibodies overnight at $4^{\circ} \mathrm{C}$. After washing with PBST (phosphate-buffered saline containing $0.05 \%$ Tween 20) for three or four times, then the membrane was incubated with the secondary antibody for $1 \mathrm{~h}$ at room temperature. Protein bands were visualized using the Odyssey Infrared Imaging System (Li-COR Biosciences). The antibodies used in this study were as follows: USP32 (sc-374465, Santa Cruz Biotechnology, 1:100), $\beta$-actin (\#81178, Santa Cruz Biotechnology, 1:1,000), SMAD2 (\#12570-1-AP, Proteintech, Wuhan, China, 1:500), p-SMAD2 (\#18338, Cell Signaling Technology, US), and FLAG (F1804, Sigma-Aldrich, US).

\section{RNA interference and SMAD2 construct}

GC cells were transfected with siRNAs or plasmids using Lipofectamine 3000 (Invitrogen; Thermo Fisher Scientific) in accordance with the manufacturer's instructions. All of siRNAs used in this study were purchased from Gene Pharma, Shanghai, China. The siRNAs were designed as follows: 5'-UUCUCCGAACGUGUCACGUdTdT -3' (si-NC, as a negative control) $5^{\prime}$-GACCUGUGGACU CUCAUAUTT-3' (si-USP32-homo-386); 5'-GGACAG UUAUAUGCACUUATT-3' (si-USP32-homo-3029). Lentiviral particles for knockdown of USP32 were packaged from GenePharma, Shanghai using above corresponding sequences (si-USP32-homo-386 and siNC). The SMAD2 expression plasmid fused a C-terminal FLAG was purchased from ViGene Bioscience, China (\#CH809987).

\section{Cas9 construction}

The Lentiviral CRISPR/Cas9 system sgRNAs were synthesized and sub-cloned by Shanghai GenePharma, China. The sgRNA sequences targeting USP32 was 5'-GGTTATTGAAGGCTCAATCCCGG-3' (sgUSP32) and a negative control 5'-TCGTACTCTA CAGCAGATGC-3' (sgNC). The MGC803 and 
SGC7901 cells were seeded in 6-well plates and infected with the Cas9 lentivirus, respectively. After puromycin selection, MGC803-sgUSP32, MGC803sgNC, SGC7901-sgUSP32 and SGC7901-sgNC were generated.

\section{Quantitative real-time PCR (qRT-PCR)}

Total RNAs from cells or tissues were extracted using TRIzol reagent (Sigma-Aldrich) and reverse transcription was conducted by the Prime script ${ }^{\mathrm{TM}} \mathrm{RT}$ Reagent kit with gDNA Eraser (Takara, Japan). The expressions of the target genes were evaluated by qRT-PCR on Applied Biosystems 7500 Fast Real-Time PCR system with Power TB Green PCR Master Mix (TaKaRa, Japan). The following primers were used for qRT-PCR assays: $\beta$-actin-qF, 5-CCTGGCACCCAGCA CAATG-3 and $\beta$-actin-qR, 5-GGGCCGGACTCGTCA TACT-3; SMAD2-qF, 5-ATGTCGTCCATCTTGCCAT TC-3 and SMAD2-qR, 5-AACCGTCCTGTTTTCTTTA GCTT-3.

\section{Patients' specimens}

The tissue microarray that contains 314 GC tumor specimens and 22 non-tumor specimens was purchased from Alenabio Company (MC6162, Xi'an, China). These tissue samples included the pathologic grade and the clinical stage. USP32 expression was assessed by immunohistochemical staining following the standard immunohistochemical procedures and the staining results were double-blindly scored. A total of 36 pairs of gastric cancer samples were gathered at Shanghai East Hospital from 2013 to 2015 with informed consents. All the samples were snap-frozen in liquid nitrogen after resection and stored at $-80^{\circ} \mathrm{C}$ prior to RNA extraction. The study for use of clinical samples was approved by the Medical Ethics Committees of Shanghai East Hospital.

\section{Animal experiments}

Male nude mice (4-5 weeks, BALB/c) were purchased from SLAC Laboratory Animal Center at Shanghai, China. All animals received standard care, and protocols for study were approved by the Medical Ethics Committee of Shanghai East Hospital. For xenograft model of gastric cancer, $2 \times 10^{6}$ stably infected cells were injected subcutaneously and bilaterally into the flank of nude mice $(n=8)$. After 21 days, the tumors were isolated and weighed. For tumor metastasis assay, the tail vein of each mouse was injected with $1.5 \times 10^{6}$ cells $(n=5)$. After 28 days, mice were euthanized and the tumor nodules on the lung surface were calculated. Hematoxylin and eosin (H\&E) of lung tissues was performed and photographed.

\section{Path scan intracellular signaling array}

A primary PathScan ${ }^{\circledR}$ Intracellular Signaling Array Kit \#12923 (Cell Signaling Technology, US) was employed to detect the intracellular signaling according to the manufacturer's instructions. The result was visualized using the Odyssey Infrared Imaging System and quantified by Image Studio software.

\section{Luciferase assay}

For reporter assays, USP32 silenced cells and negative control cells were plated in 24-well plates and transfected with 2 ug SMAD-Luc reporter plasmid and $50 \mathrm{ng}$ of pRL-SV40 Renilla luciferase construct. $24 \mathrm{~h}$ after transfection, SMAD Response Element (SRE) activity was measured using a dual luciferase assay kit (Promega, USA).

\section{Flow cytometry analysis}

The Annexin V-FITC/PI apoptosis detection kit (KGA-107, KeyGEN, China) was used to detect the cell apoptosis following the manufacturer's instructions. Data was analyzed by flow cytometer and the experiment was repeated three times.

\section{Statistical analysis}

Quantitative data are presented as the mean \pm S.E.M. or mean \pm S.D., statistical significance was determined by Student's t-test or $X^{2}$ test. Statistical significance is represented in figures by: ${ }^{*} P<0.05$; ** $P<0.01$.

\section{Results}

\section{Silencing of USP32 inhibits GC cell growth and migration in vitro}

USP32 expression was knocked down in MGC803 and SGC7901 cells by transient transfection with siRNAs against USP32, and result of western blot analysis indicated that siUSP32-386 and siUSP323029 markedly decreased USP32 expression (Figure 1A). Subsequently, CCK-8 assay was employed to investigate the effect of USP32 knockdown on cell growth. The results demonstrated that transient silencing of USP32 in MGC803 and SGC7901 cells significantly reduced cell growth rate (Figure 1A). To further confirm the function of USP32 in GC cells, stable cell lines were established by two approaches. One was lentivirus-mediated USP32 knockdown with shUSP32-386, the other was lentivirus-mediated depletion of USP32 based on CRISPR/Cas9 technique. As shown in Figure 1B, growth curve results revealed that stable silencing or depletion of USP32 inhibited the proliferation capacity of GC cells. Consistently, downregulation of USP32 also led to an inhibitory effect on colony formation of those stable cells (Figure 
1C). In addition, we observed that cell migration ability was decreased in USP32 downregulated GC cells by a Transwell chamber analysis (Figure 1D). These collective data suggested the critical roles of USP32 in promoting cell proliferation and migration in vitro.

\section{Silencing of USP32 attenuates tumor growth and metastasis of GC cells in vivo}

To further address the effect of USP32 on tumor growth in vivo, a tumor xenograft model was established and cells with stable downregulation of USP32 and corresponding control cells were subcutaneously injected into 4-week-old male nude mice ( $\mathrm{n}=8$ per group), and tumor tissue were harvested 3 weeks after injection. As expected, the tumors in USP32 downregulation group exhibited lower mass and smaller size than those in control group (Figure 2A), suggesting that USP32 is required for tumorigenicity of GC cells in vivo. Moreover, pulmonary metastasis assays were performed and the results showed that downregulation of USP32 markedly decreased metastatic foci on lung surfaces (Figure 2B). These data further confirmed the oncogenic role of USP32 in animal models.
A

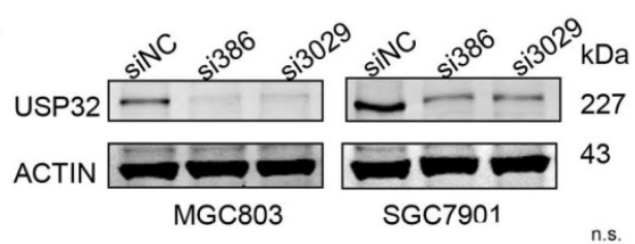

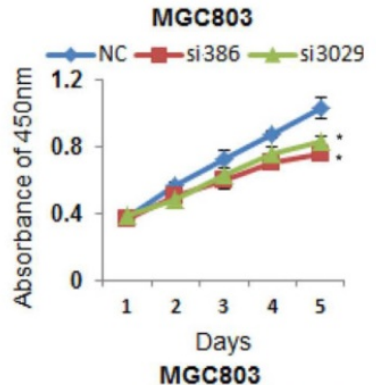

MGC803

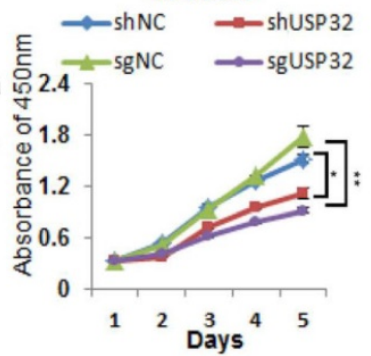

C
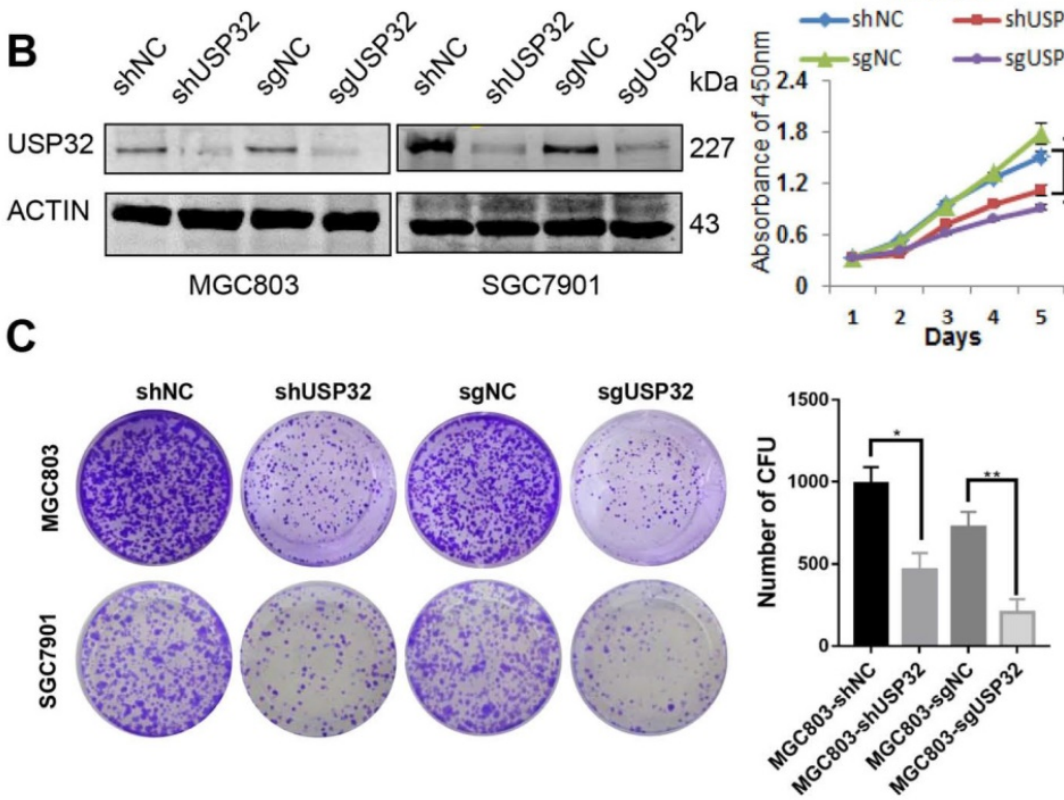
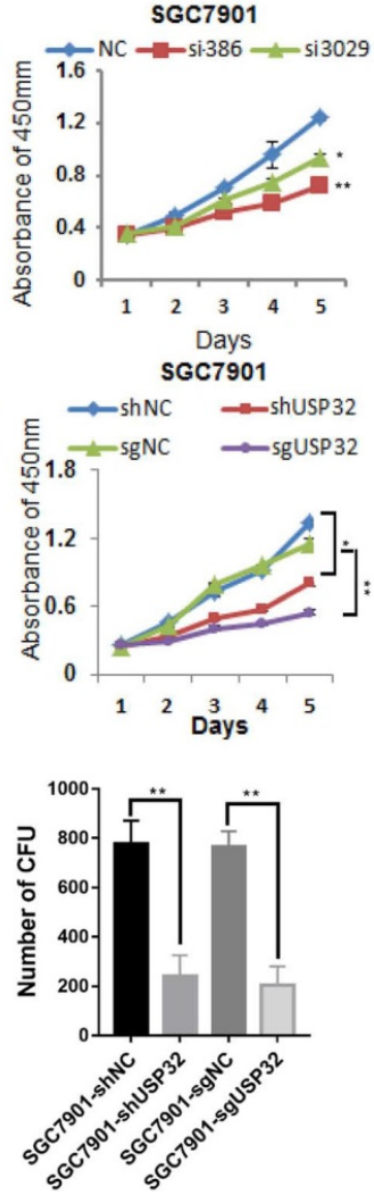

\section{D}
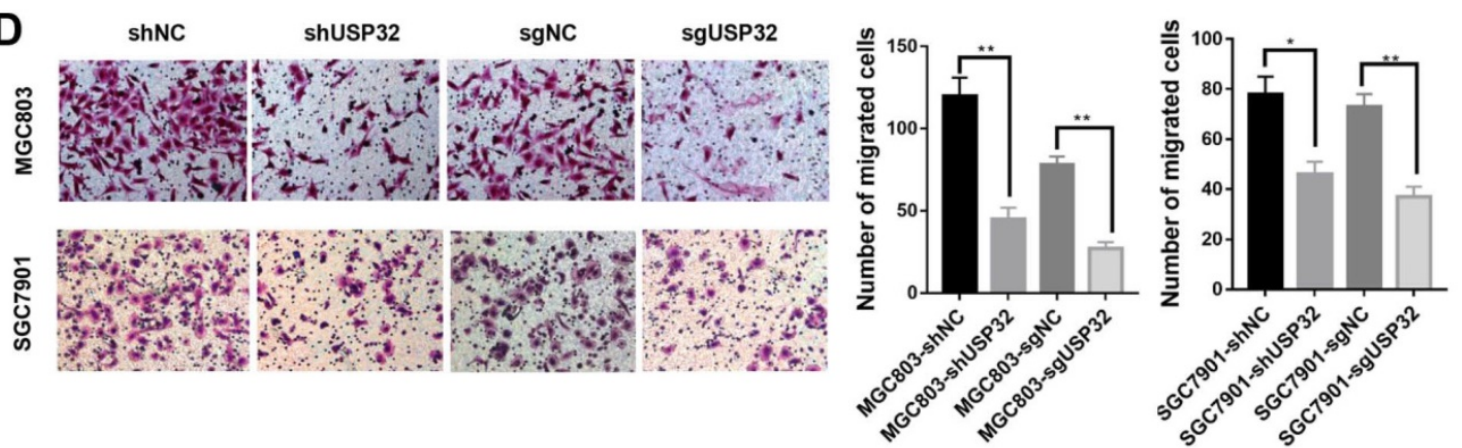

Figure 1. Knockdown of USP32 inhibited GC cell growth and migration in vitro. (A) The knockdown efficiency of USP32 was determined by western blotting (siUSP32-386 and siUSP32-3029). CCK8 assays were performed in the cells as indicated. (B) Western blot and CCk8 assays were performed in stable cell lines infected with lentivirus (LV-shUSP32 or control LV-shNC; LV-sgUSP32 or control LV-sgNC). (C) Cell colonies were stained and counted two weeks after plating GC cells as indicated into 6-well plates. (D) Knockdown or depletion of USP32 decreased the number of migrated cells in MGC803 and SGC7901 cells. $* P<0.05$, $* * P<0.01$. 

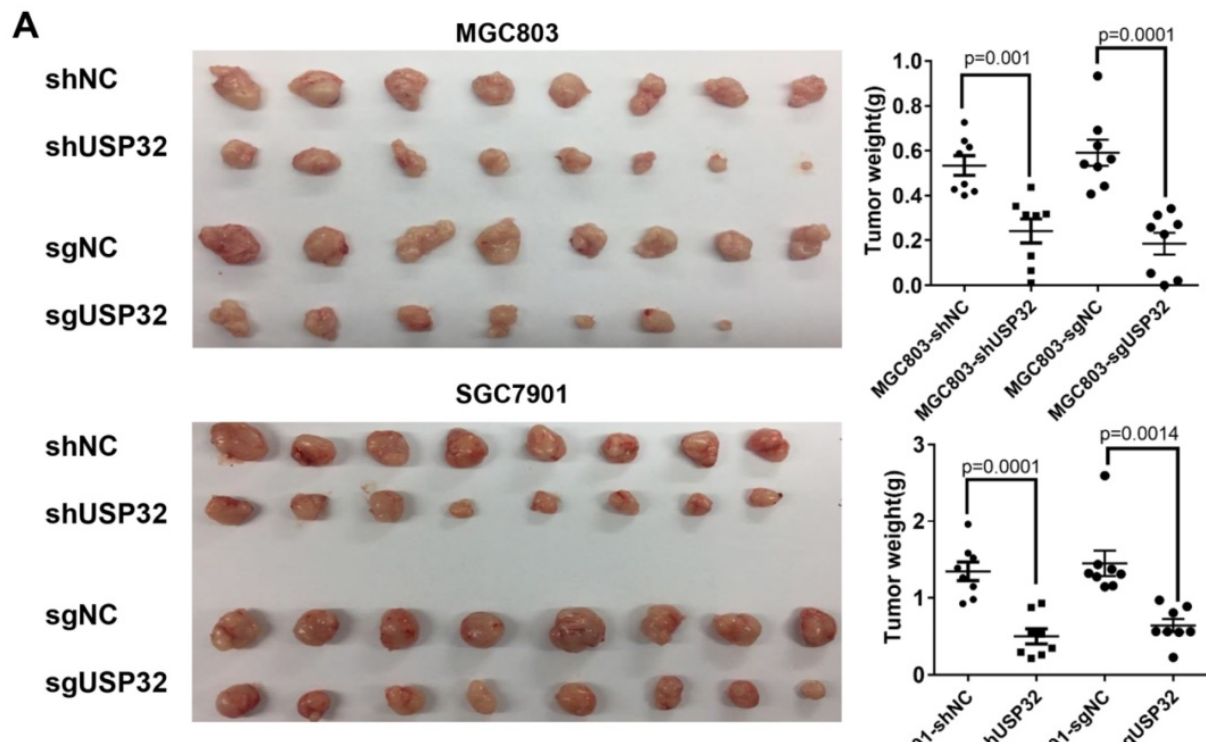

B
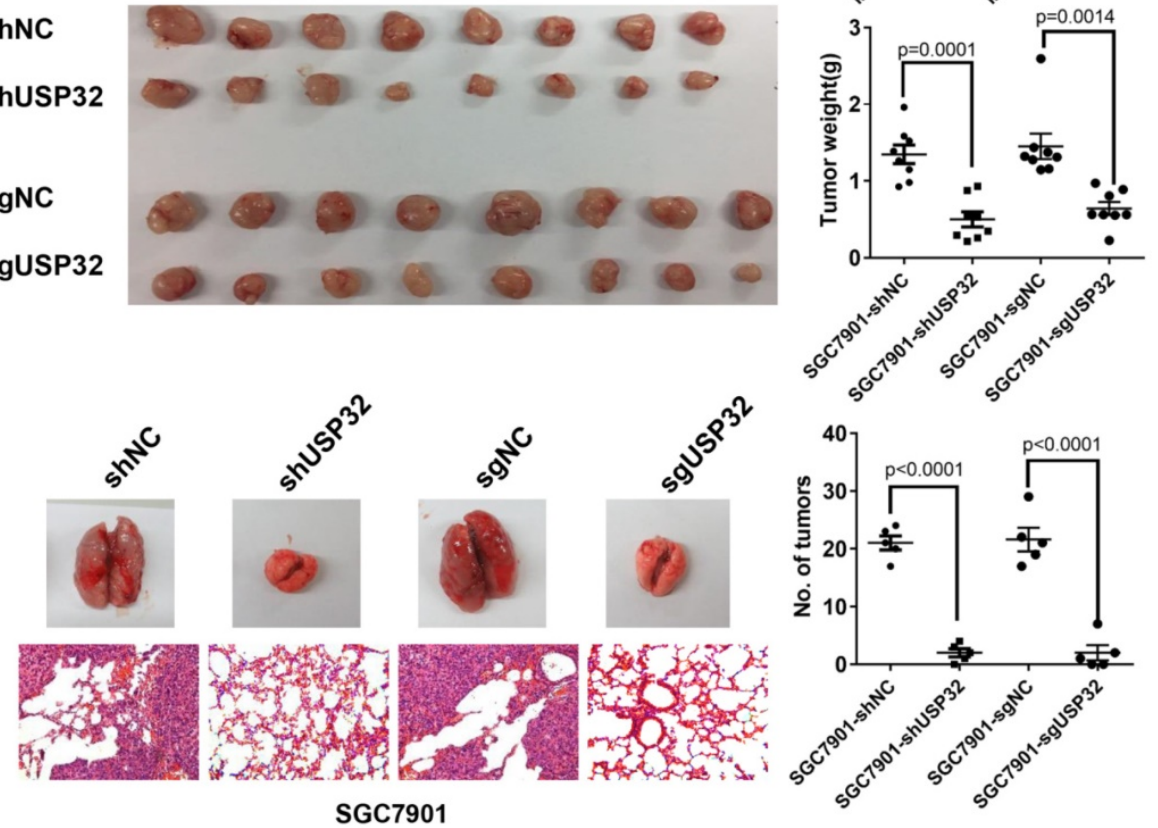

Figure 2. Knockdown of USP32 suppressed the tumorigenicity and metastasis of GC cells in vivo. (A) Knockdown of USP32 decreased the tumorigenicity of MGC803 and SGC7901 cells in nude mice. MGC803 and SGC7901 cells stably expressing shUSP32 or sgUSP32 were subcutaneously injected into nude mice. Mice were sacrificed after 3 weeks and tumor weights were measured. Values are expressed as mean \pm SD $(n=8)$. (B) Knockdown of USP32 inhibited lung metastasis of SGC7901 cells. Lung metastatic tumor nodules on lung surface were counted and lung sections were hematoxylin eosin (HE) stained $(n=5, M a g n i f i c a t i o n: \times 200)$.

\section{USP32 is upregulated in gastric cancer tissues and is related to tumor stage}

To explore the expression pattern of USP32 in GC, we performed an immunohistochemical (IHC) analysis using USP32 specific antibody in a human gastric cancer tissue microarray, which contains 314 tumor samples and 22 non-tumor tissues. As shown in Figure 3A, the expression level of USP32 was graded by intensity of IHC staining. The strong staining of USP32 was far more frequently observed in gastric cancer tissues $(197 / 314,62.7 \%)$ than in paracarcinoma normal tissues $(5 / 22,22.7 \%)$. Furthermore, there was a significant correlation between USP32 expression and $\mathrm{T}$ staging (Figure 3B, $P<0.001$ ). Consistent with our IHC results, high expression of USP32 was found in tumor tissues by analyzing TCGA database using GEPIA online tools (http:// gepia.cancer-pku.cn/) (Figure 3C) and performing qRT-PCR assay in 36 pairs of gastric cancer samples (Figure 3D). Additionally, by analyzing Kaplan-Meier Plotter datasets (http://kmplot.com/analysis/index. php? $\mathrm{p}=$ service\&cancer=gastric), USP32 expression was shown to be significantly correlated with poor prognosis of gastric cancer patients (Figure 3E). These results strongly suggested that USP32 is frequently overexpressed in GC and could act as a biomarker for GC.

\section{USP32 regulates SMAD2 expression in GC cells}

A PathScan ${ }^{\circledR}$ intracellular signaling array was employed to elucidate the downstream effectors of USP32 in GC cells. As shown in Figure 4A, phosphorylated (p-) SMAD2 level was significantly reduced in USP32 silenced MGC803 cells. To validate this result, dual luciferase reporter assays were performed with SMAD2/3 response element reporter plasmid, and the results indicated that the luciferase activity was markedly decreased with the absence of USP32 (Figure 4B). qRT-PCR results demonstrated that USP32 downregulation had no effect on SMAD2 transcription (Figure 4C). Interestingly, when we detected the expression level of p-SMAD2 via western 
blot analysis, we found that the level of p-SMAD2 was decreased accompanied by a reduction of total protein level of SMAD2 in USP32 downregulated cells (Figure 4D). Accordingly, GC cells were treated with cycloheximide (CHX), a reagent for blocking de novo protein synthesis. Results from western blots indicated that downregulation of USP32 dramatically decreased the SMAD2 protein level after protein synthesis was inhibited (Figure $4 \mathrm{E}$ ). As the same time, the decrease in SMAD2 protein level induced by USP32 downregulation could be rescued by the proteasome inhibitor MG132 (Figure 4F). These results suggested that USP32 may regulate SMAD2 expression by enhancing protein stabilization of SMAD2.

\section{SMAD2 restores USP32 knockdown induced growth and migration defects}

To investigate the functional relevance of USP32 and SMAD2, we overexpressed SMAD2 together with knockdown of USP32 in MGC803 cells (Figure 5A). The CCK8 assays revealed that overexpression of SMAD2 significantly attenuated the inhibitory effect on cell proliferation caused by knockdown of USP32 (Figure 5B). Similarly, Transwell chamber assays also proved that knockdown of USP32 decreased the number of migrated cells while overexpression of SMAD2 compensated this phenotype (Figure 5C). Therefore, these data indicated that SMAD2 could restore the oncogenic effects of USP32 in GC cell proliferation and migration.

A

weak staining

strong staining
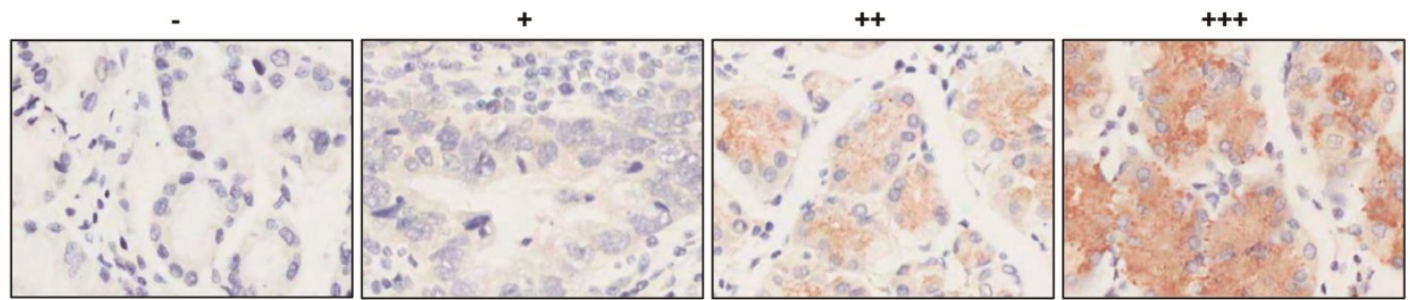

B
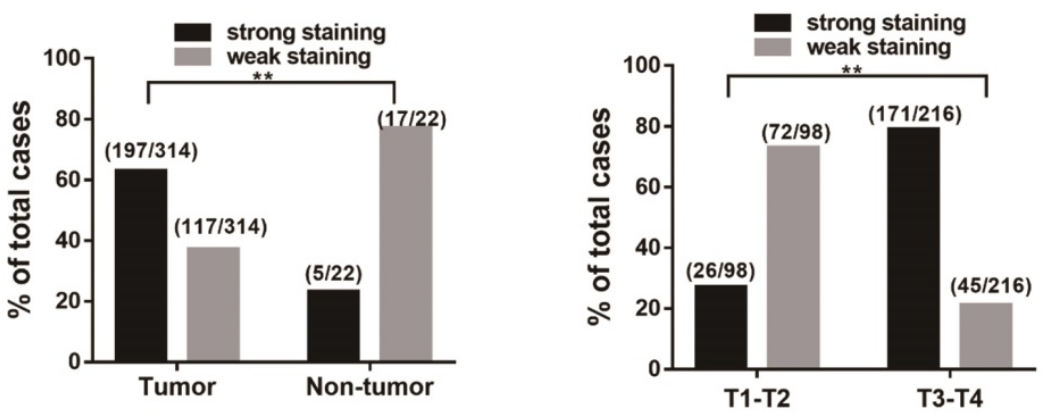

C

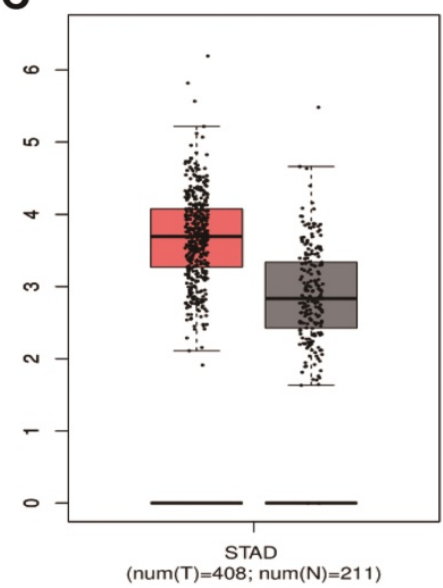

D

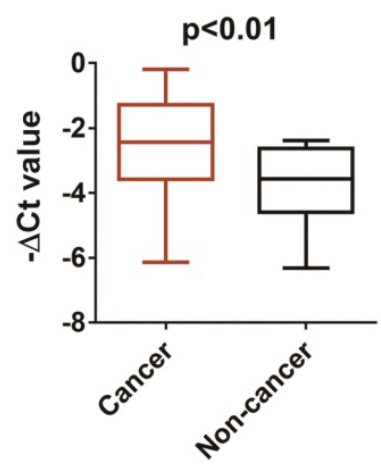

E

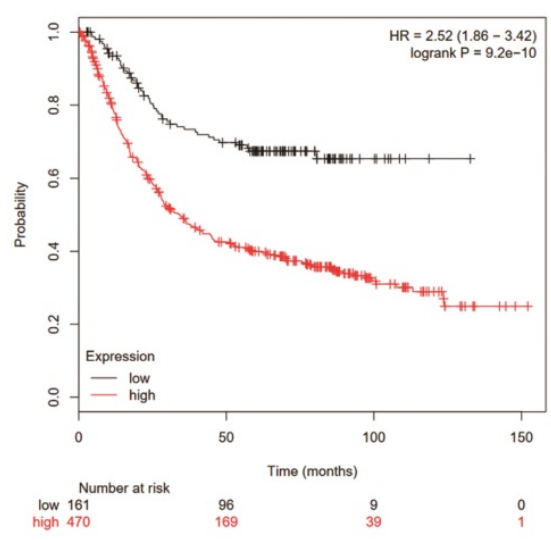

Figure 3. The expression pattern of USP32 in gastric cancer tissues. (A) The expression of USP32 was determined by immunohistochemical staining on sections of gastric cancer tissues. "-" (negative); “+” (slightly positive) “++" (moderately positive); “+++” (strongly positive). Magnification: $\times 200$. (B) The ratio for strong stating (++, +++) and for weak staining $(-,+)$ in tumor and non-tumor tissues (left) and in T1-T2 stage and T3-T4 stage (right). **P<0.01. (C) The expression of USP32 from TCGA database. (D) qRT-PCR was conducted to explore the expression of USP32 in 36 pairs of gastric cancer samples. (E) Kaplan-Meier plot displayed the overall survival patients with high (red line; $n=470$ ) and low (black line; $n=96 ; P<0.01$, Log-rank test) expression of USP32. 


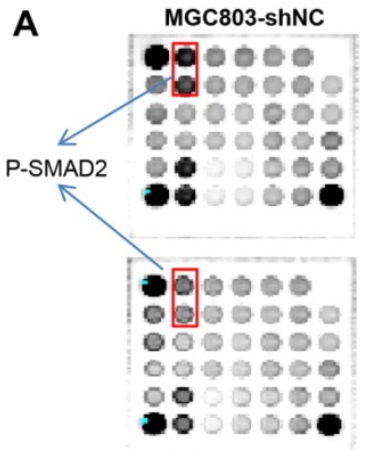

MGC803-shUSP32

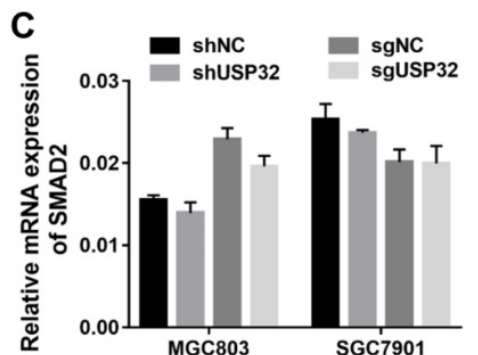

E

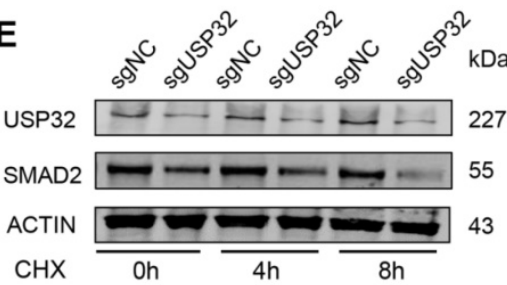

B
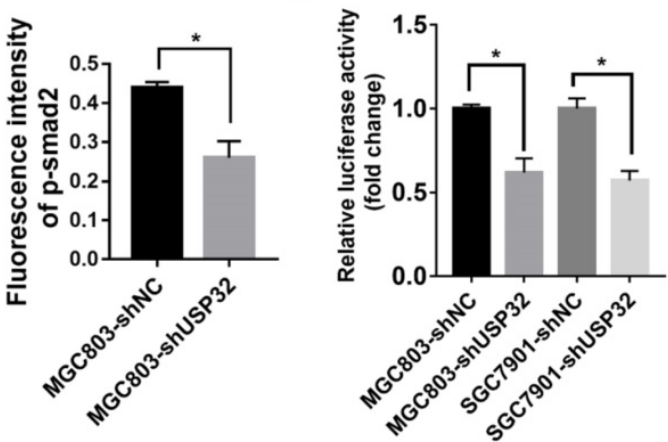

D

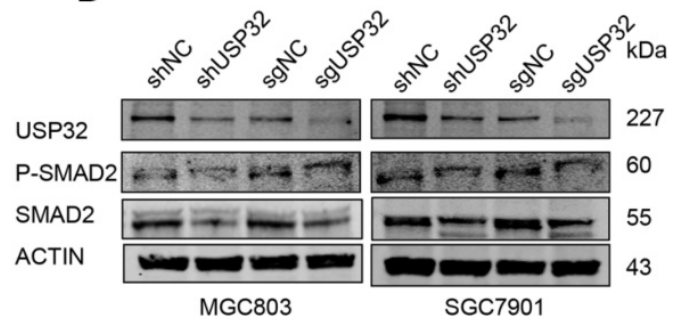

$\mathbf{F}$

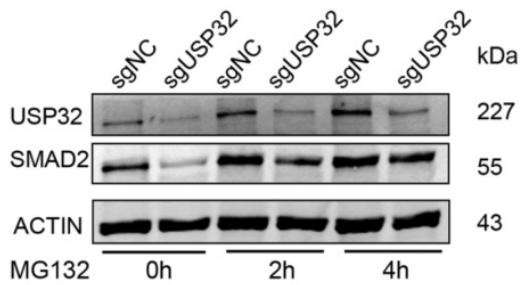

Figure 4. Effects of USP32 downregulation on the expression of SMAD2 in GC cells. (A) Intracellular signaling array was performed in MGC803 cells. The P-SMAD2 dots was marked with rectangles and quantified by Image Studio Version 3.1. (B) Relative luciferase activity of MGC803 or SGC7901 cells in which USP32 was stably downregulated. SMAD2/3 response element (SRE) luciferase reporter and a Renilla luciferase plasmid were co-transfected. $* P<0.05$. (C) The mRNA level of SMAD2 was detected by qRT-PCR in MGC803 and SGC7901 cells. The data were normalized with the $\beta$-actin mRNA. (D) Western blot analysis was used to confirm the p-SMAD2 and SMAD2 protein level in MGC803 and SGC7901 cells as indicated. (E) MGC803 cells stably expressing control (sgNC) or USP32 sgRNAs were treated with CHX, and collected at the indicated times, then western blot analysis was performed. (F) MGC803 cells stably expressing control (sgNC) or USP32 sgRNAs were treated with MG132, and collected at the indicated times, then western blot analysis was performed.

A

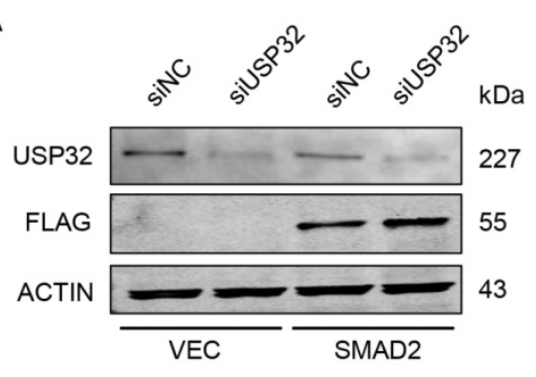

C

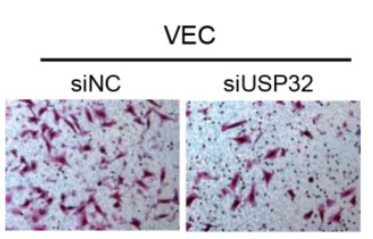

B
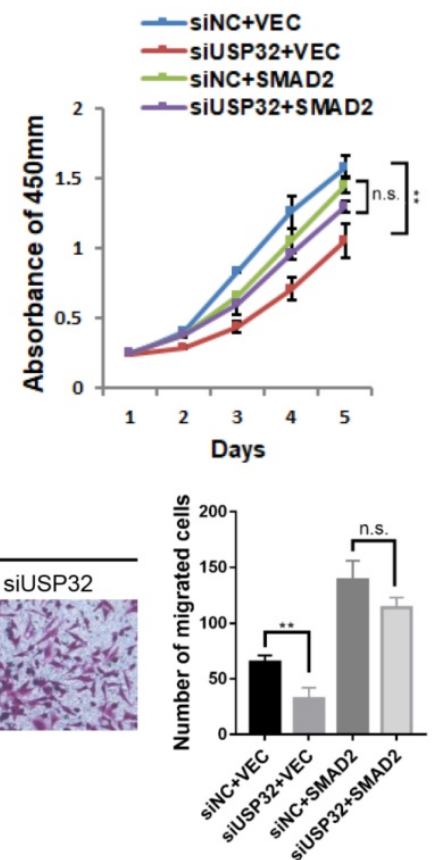

Figure 5. SMAD2 rescued phenotypic defects in cell growth and migration caused by downregulation of USP32. (A) Western blot analysis was performed to confirm the overexpression of SMAD2 and the silencing of USP32. (B) Silencing of USP32 significantly inhibited cell growth in MGC803 cells while overexpressed SMAD2 attenuated the inhibitory effect. (C) Transwell assays were used to detect the effects of USP32 and SMAD2 expression on cell migratory ability in MGC803 cells. 


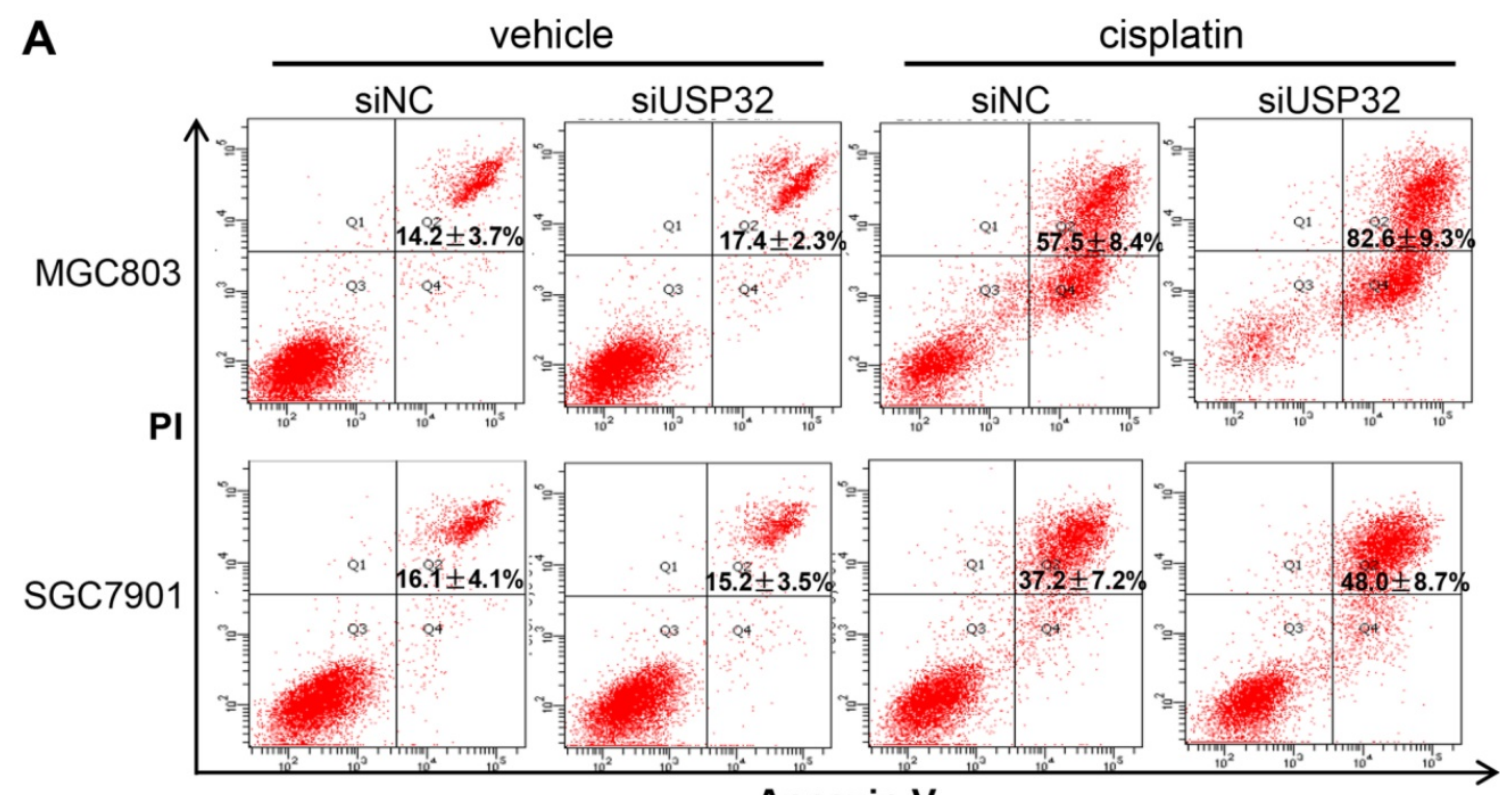

\section{Annexin V}

B

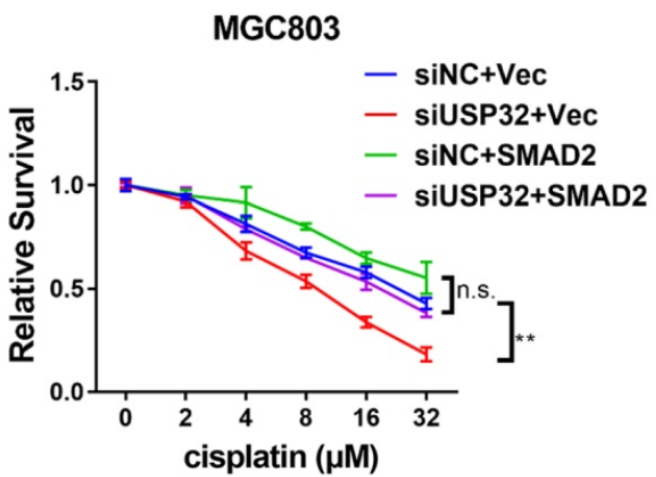

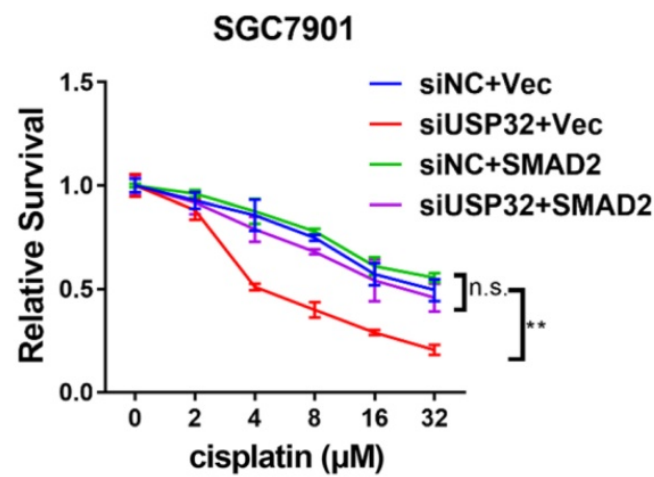

Figure 6. USP32 downregulation sensitized GC cells to cisplatin. (A) $48 \mathrm{~h}$ after cisplatin treatments, flow cytometry analysis was performed in MGC803 and SGC7901 cells transfected with si-NC or si-USP32. (B) Cells with USP32 knockdown or SMAD2 overexpression were treated with cisplatin as indicated concentrations for 48 h, and then cell viabilities were determined by CCK8 assays.

\section{USP32 confers drug resistance of GC cells}

It has been reported that TGF $\beta /$ SMAD signaling pathway involved in cisplatin resistance of tumor cells $[23,24]$. Considering USP32 regulates SMAD2 expression, we attempted to elucidate whether USP32 could mediate this process. By flow cytometric analyses, we demonstrated that cisplatin-induced cell death was increased in USP32 knockdown cells compared with control cells (Figure 6A). Furthermore, knockdown of USP32 attenuated the resistance of GC cells to cisplatin in CCK8 assays, whereas overexpression of SMAD2 partially rescued the defect of USP32 silenced GC cells in cisplatin resistance (Figure 6B), suggesting that USP32 may confer chemoresistance in GC.

\section{Discussion}

Gastric cancer still remains the third leading cause of cancer-related death despite the advances that have been made in diagnosis and treatment [25].
Due to the limited therapeutic options, late diagnosis and uncontrolled recurrence, the 5-year survival rate for GC patients is very low. Therefore, it is urgent to develop new and more effective therapeutic targets for GC. In the present study, we identified USP32, a new ubiquitin-specific protease, as an oncogene involved in GC cell growth and metastasis.

Our data firstly demonstrate that USP32 downregulation significantly inhibited GC cell proliferation and migration in vitro, as well as in a mouse model. These results are consistent with previous report in breast cancer and small lung cancer $[18,19]$, suggesting that USP32 may function as an oncogene in tumors. Meanwhile, we also show that USP32 was upregulated in GC tissues and high expression of USP32 was related to low overall survival of GC patients and high $\mathrm{T}$ staging. The findings raise the possibility that USP32 might serve as a potential biomarker and therapeutic target for GC. 
Next, we provide evidence that knockdown of USP32 decreased the expression of SMAD2. SMAD2, a vital mediator of TGF- $\beta$ signaling pathway, is subjected to regulation in changing subcellular localization and transcriptional response [26]. SMAD2 has been reported to be abnormal expression in many cancers. Tang et al reported that profilin-2 (Pfn2) enhances Smad2/Smad3 expression, and high SMAD expression correlates with poor outcome of lung cancer patients [27]. In addition, several investigations have indicated the oncogenic roles of SMAD2 in pancreatic cancer, gastric cancer, and prostate cancer [28-30]. However, it is controversial for the functional roles of SMAD2 in cancer. In other reports, SMAD2 was shown to exert tumor suppressor function during tumorigenesis [31,32]. To date, the exact functions and regulatory mechanisms of SMAD2 in cancer are complicated and not fully understood. In our case, we found the protein expression of SMAD2 is no longer affected by USP32 after treatment with proteasome inhibitor MG132. This suggests that SMAD2 is regulated by USP32 through proteasome pathway, although we did not find any direct interaction between SMAD2 and USP32 via coimmunoprecipitation experiments (Data not shown). The rescue experiments also indicate that SMAD2 severs as the downstream of USP32 and could restore at least partial defects in growth and migration of USP32 silenced cells.

Many researches have shown deubiquitylating enzymes participate in chemoresistance $[33,34]$. Song et al reported that deubiquitylating enzyme Rpn11 can enhance the chemosensitivity of bortezomib in multiple myeloma cells [35]. Other than that, Qin et al demonstrated that silencing of USP37 can reduce resistance to cisplatin-targeting therapies in breast cancer [36]. In this regard, we also observed that USP32 downregulation could decrease the cisplatinresistance in GC cells, suggesting the involvement of USP32 in drug resistance. In the meanwhile, overexpression of SMAD2 can enhance the resistance to cisplatin in USP32 silenced cells. These observations further support the previous proposal that SMAD2 functions as downstream of USP32 in GC.

In conclusion, our findings provide a new insight into the expression and function of USP32 in cancer. SMAD2 may mediate USP32-induced cell growth, metastasis and chemoresistance. Targeting USP32 might be a potential therapeutic strategy for GC.

\section{Acknowledgments}

This study was supported by grants from the National Key Research and Development Program of
China (No. 2017YFC1308900), National Natural Science Foundation of China (No. 81772567), Key Disciplines Group Construction Project of Pudong Health Bureau of Shanghai (No.PWZxq2017-13), and the Outstanding Clinical Discipline Project of Shanghai Pudong (No.PWYgy2018-02).

\section{Competing Interests}

The authors have declared that no competing interest exists.

\section{References}

1. Yau R, Rape M. The increasing complexity of the ubiquitin code. Nat Cell Biol. 2016; 18: 579-86.

2. Edmonds MJ, Carter RJ, Nickson CM, Williams SC, Parsons JL. Ubiquitylation-dependent regulation of NEIL1 by Mule and TRIM26 is required for the cellular DNA damage response. Nucleic Acids Res. 2017; 45: 726-38.

3. Rape M. Ubiquitylation at the crossroads of development and disease. Nat Rev Mol Cell Biol. 2018; 19: 59-70.

4. Aksentijevich I, Zhou Q. NF-kappaB Pathway in Autoinflammatory Diseases: Dysregulation of Protein Modifications by Ubiquitin Defines a New Category of Autoinflammatory Diseases. Front Immunol. 2017; 8: 399.

5. Graner E, Tang D, Rossi S, Baron A, Migita T, Weinstein LJ, et al. The isopeptidase USP2a regulates the stability of fatty acid synthase in prostate cancer. Cancer Cell. 2004; 5: 253-61.

6. Vinas-Castells R, Beltran M, Valls G, Gomez I, Garcia JM, Montserrat-Sentis B, et al. The hypoxia-controlled FBXL14 ubiquitin ligase targets SNAIL1 for proteasome degradation. J Biol Chem. 2010; 285: 3794-805.

7. Nijman SM, Luna-Vargas MP, Velds A, Brummelkamp TR, Dirac AM, Sixma TK, et al. A genomic and functional inventory of deubiquitinating enzymes. Cell. 2005; 123: 773-86.

8. Pal A, Donato NJ. Ubiquitin-specific proteases as therapeutic targets for the treatment of breast cancer. Breast Cancer Res. 2014; 16: 461.

9. Zhang Z, Fan Y, Xie F, Zhou H, Jin K, Shao L, et al. Breast cancer metastasis suppressor OTUD1 deubiquitinates SMAD7. Nat Commun. 2017; 8: 2116.

10. He L, Liu X, Yang J, Li W, Liu S, Liu X, et al. Imbalance of the reciprocally inhibitory loop between the ubiquitin-specific protease USP43 and EGFR/PI3K/AKT drives breast carcinogenesis. Cell Res. 2018; 28: 934-51.

11. Diefenbacher ME, Popov N, Blake SM, Schulein-Volk C, Nye E, Spencer-Dene $\mathrm{B}$, et al. The deubiquitinase USP28 controls intestinal homeostasis and promotes colorectal cancer. J Clin Invest. 2014; 124: 3407-18.

12. Gennaro VJ, Stanek TJ, Peck AR, Sun Y, Wang F, Qie S, et al. Control of CCND1 ubiquitylation by the catalytic SAGA subunit USP22 is essential for cell cycle progression through G1 in cancer cells. Proc Natl Acad Sci U S A. 2018; 115: E9298-E307.

13. Kiran S, Dar A, Singh SK, Lee KY, Dutta A. The Deubiquitinase USP46 Is Essential for Proliferation and Tumor Growth of HPV-Transformed Cancers. Mol Cell. 2018; 72(e5): 823-35.

14. Sun J, Li T, Zhao Y, Huang L, Sun H, Wu H, et al. USP10 inhibits lung cancer cell growth and invasion by upregulating PTEN. Mol Cell Biochem. 2018; 441: $1-7$.

15. Chen X, Wang C, Liao K, Zhou S, Cao L, Chen J, et al. USP17 Suppresses Tumorigenesis and Tumor Growth through Deubiquitinating AEP. Int J Biol Sci. 2019; 15: 738-48.

16. Luo $\mathrm{K}, \mathrm{Li} \mathrm{Y}$, Yin $\mathrm{Y}$, $\mathrm{Li} \mathrm{L}, \mathrm{Wu} \mathrm{C}$, Chen $\mathrm{Y}$, et al. USP49 negatively regulates tumorigenesis and chemoresistance through FKBP51-AKT signaling. EMBO J. 2017; 36: 1434-46.

17. Paulding CA, Ruvolo $M$, Haber DA. The Tre2 (USP6) oncogene is a hominoid-specific gene. Proc Natl Acad Sci U S A. 2003; 100: 2507-11.

18. Akhavantabasi S, Akman HB, Sapmaz A, Keller J, Petty EM, Erson AE. USP32 is an active, membrane-bound ubiquitin protease overexpressed in breast cancers. Mamm Genome. 2010; 21: 388-97.

19. Hu W, Wei H, Li K, Li P, Lin J, Feng R. Downregulation of USP32 inhibits cell proliferation, migration and invasion in human small cell lung cancer. Cell Prolif. 2017; 50.

20. Zhou F, Xie F, Jin K, Zhang Z, Clerici M, Gao R, et al. USP4 inhibits SMAD4 monoubiquitination and promotes activin and BMP signaling. EMBO J. 2017; 36: 1623-39.

21. Agricola E, Randall RA, Gaarenstroom T, Dupont S, Hill CS. Recruitment of TIF1gamma to chromatin via its PHD finger-bromodomain activates its ubiquitin ligase and transcriptional repressor activities. Mol Cell. 2011; 43: 85-96.

22. Kavsak P, Rasmussen RK, Causing CG, Bonni S, Zhu H, Thomsen GH, et al. Smad7 binds to Smurf2 to form an E3 ubiquitin ligase that targets the TGF beta receptor for degradation. Mol Cell. 2000; 6: 1365-75.

23. Oshimori N, Oristian D, Fuchs E. TGF-beta promotes heterogeneity and drug resistance in squamous cell carcinoma. Cell. 2015; 160: 963-76. 
24. Zhu H, Gu X, Xia L, Zhou Y, Bouamar H, Yang J, et al. A Novel TGFbeta Trap Blocks Chemotherapeutics-Induced TGFbeta1 Signaling and Enhances Their Anticancer Activity in Gynecologic Cancers. Clinical cancer research : an official journal of the American Association for Cancer Research. 2018; 24: 2780-93.

25. Torre LA, Bray F, Siegel RL, Ferlay J, Lortet-Tieulent J, Jemal A. Global cancer statistics, 2012. CA Cancer J Clin. 2015; 65: 87-108.

26. Ikushima H, Miyazono K. TGFbeta signalling: a complex web in cancer progression. Nat Rev Cancer. 2010; 10: 415-24.

27. Tang YN, Ding WQ, Guo XJ, Yuan XW, Wang DM, Song JG. Epigenetic regulation of Smad2 and Smad3 by profilin-2 promotes lung cancer growth and metastasis. Nat Commun. 2015; 6: 8230.

28. Chen K, Cheng L, Qian W, Jiang Z, Sun L, Zhao Y, et al. Itraconazole inhibits invasion and migration of pancreatic cancer cells by suppressing TGF-beta/SMAD2/3 signaling. Oncol Rep. 2018; 39: 1573-82.

29. Zhang W, Li Y. miR-148a downregulates the expression of transforming growth factor-beta2 and SMAD2 in gastric cancer. Int J Oncol. 2016; 48: 1877-85.

30. Kou B, Liu W, Zhao W, Duan P, Yang Y, Yi Q, et al. Thymoquinone inhibits epithelial-mesenchymal transition in prostate cancer cells by negatively regulating the TGF-beta/Smad2/3 signaling pathway. Oncol Rep. 2017; 38: 3592-8.

31. Zhao Z, Fan X, Jiang L, Xu Z, Xue L, Zhan Q, et al. miR-503-3p promotes epithelial-mesenchymal transition in breast cancer by directly targeting SMAD2 and E-cadherin. J Genet Genomics. 2017; 44: 75-84.

32. Yeh HW, Hsu EC, Lee SS, Lang YD, Lin YC, Chang CY, et al. PSPC1 mediates TGF-beta1 autocrine signalling and Smad2/3 target switching to promote EMT, stemness and metastasis. Nat Cell Biol. 2018; 20: 479-91.

33. Lan X, Zhao C, Chen X, Zhang P, Zang D, Wu J, et al. Platinum pyrithione induces apoptosis in chronic myeloid leukemia cells resistant to imatinib via DUB inhibition-dependent caspase activation and Bcr-Abl downregulation. Cell Death Dis. 2017; 8: e2913.

34. Didier R, Mallavialle A, Ben Jouira R, Domdom MA, Tichet M, Auberger P, et al. Targeting the Proteasome-Associated Deubiquitinating Enzyme USP14 Impairs Melanoma Cell Survival and Overcomes Resistance to MAPK-Targeting Therapies. Mol Cancer Ther. 2018; 17: 1416-29.

35. Song Y, Li S, Ray A, Das DS, Qi J, Samur MK, et al. Blockade of deubiquitylating enzyme Rpn11 triggers apoptosis in multiple myeloma cells and overcomes bortezomib resistance. Oncogene. 2017; 36: 5631-8.

36. Qin T, Li B, Feng X, Fan S, Liu L, Liu D, et al. Abnormally elevated USP37 expression in breast cancer stem cells regulates stemness, epithelial-mesenchymal transition and cisplatin sensitivity. J Exp Clin Cancer Res. 2018; 37: 287. 